\title{
A direct approach to the discounted penalty function
}

\author{
Hansjörg Albrecher*, Hans U. Gerber ${ }^{\dagger}$ and Hailiang Yang ${ }^{\ddagger}$
}

\begin{abstract}
This paper provides a new and accessible approach to establishing certain results concerning the discounted penalty function. The direct approach consists of two steps. In the first step, closed form expressions are obtained in the special case where the claim amount distribution is a combination of exponential distributions. A rational function is useful in this context. For the second step, one observes that the family of combinations of exponential distributions is dense. Hence, it suffices to reformulate the results of the first step to obtain general results. The surplus process has downward and upward jumps, modeled by two independent compound Poisson processes. If the distribution of the upward jumps is exponential, a series
\end{abstract}

*Hansjörg Albrecher, Ph.D., is a Professor of Actuarial Science, Faculty of Business and Economics, University of Lausanne, CH-1015 Lausanne, Switzerland. e-mail: hansjoerg.albrecher@unil.ch

${ }^{\dagger}$ Hans U. Gerber, A.S.A., Ph.D., Distinguished Visiting Professor at the University of Hong Kong, is an Honorary Professor of Actuarial Science, Faculty of Business and Economics, University of Lausanne, CH-1015 Lausanne, Switzerland, e-mail: hgerber@unil.ch.

${ }^{\ddagger}$ Hailiang Yang, A.S.A., Ph.D., is a Professor, Department of Statistics and Actuarial Science, The University of Hong Kong, Pokfulam Road, Hong Kong, e-mail: hlyang@hkusua.hku.hk 
of new results can be obtained with ease. Subsequently, certain results of Gerber and Shiu (1998) can be reproduced. The two step approach is also applied when an independent Wiener process is added to the surplus process. Certain results are related to Zhang et al. (2010), which uses different methods.

\section{Introduction}

This paper provides a new and accessible approach to establishing certain results concerning the discounted penalty function. The method consists of two steps. In the first step, results are derived for the case where the claim amount distribution is a combination of exponential distributions. A rational function is a handy tool in this context. The second step is based on the observation that any claim amount distribution can be obtained as a limit of a sequence of combinations of exponential distributions. Thus it suffices to translate in general terms the results of the first step, in order to obtain results for an arbitrary claim amount distribution. The approach of this paper is partly inspired by Dufresne and Gerber (1989), which features the first step in the special case of the probability of ruin function.

The paper considers a model for the surplus process with downward and upward jumps, given by two independent compound Poisson processes. It is noted that the classical model with deterministic premiums can be retrieved as a limit. The two step approach is particularly fruitful in the case where the upward jumps are exponentially distributed. The corresponding results are believed to be new, and several of the results in Gerber and Shiu (1998) can be found as limits. However, it should be mentioned 
that in Gerber and Shiu (1998), the penalty may also depend on the surplus prior to ruin, which is not the case in this paper. In the last section, the two step approach is applied to the model, where an independent Wiener process is added. Again, some of the results are new, and some are somewhat related to results of Zhang et al. (2010), which uses different methods.

In the literature, sophisticated methods have been developed and applied, such as renewal theory, Laplace transforms, and deep analytical tools. In contrast, the two step approach of this paper appears simple.

In the following, we give an incomplete account of the literature of two sided jump models. Ruin theory for a compound Poisson risk model with two-sided jumps is a classical object of study, see for instance Segerdahl (1939) and Cramér (1955). Boucherie and Boxma (1996) noticed in a queueing context that upward jumps can be interpreted as an increase of interarrival times (during which the premiums are collected with constant intensity) in a risk model with negative jumps only. Hence, quantities that are invariant with respect to scaling of the time axis can equivalently be obtained from the corresponding renewal model with appropriately adjusted interarrival times and negative jumps. In particular, a Pollaczek-Khintchine formula for the ruin probability for the model with two-sided jumps was obtained in Boucherie et al. (1997). If one is interested in time-dependent quantities such as the time of ruin, the analysis is usually more delicate. Based on martingale techniques, some first-exit problems for compound Poisson processes and, more generally, for Lévy processes with two-sided jumps were recently, among others, studied by Perry et al. (2002), Kou and Wang (2003), Jacobsen (2005) and Xing et al. (2008) under certain types of assumptions on the jump distributions. For related results on explicit Wiener-Hopf factorization for classes of 
Levy models with two-sided jumps and applications to mathematical finance, see e.g. Levendorskii (2004), Lewis and Mordecki (2008) and Asmussen et al. (2008). Cai et al. (2009) apply results on two-sided exponential jumps to the pricing of perpetual American put options.

Risk models without a deterministic premium component, where both the premium income and the aggregate claim process are modelled by (independent) compound Poisson processes, recently were investigated in several papers. Temnov (2004) compares the resulting ruin probabilities with the ones of the classical risk model. Defective renewal equations for the discounted penalty function in such models were studied in Bao (2006), Labbé and Sendova (2009) and Zhang et al. (2010). See also Schmidli (2010) for a general approach using a change of measure. For an extension to renewal models see Zhang and Yang (2010).

\section{The two-sided jumps model}

Let $U(t)$ denote the surplus of a company at time $t$. We model the surplus process as

$$
U(t)=u+S(t), \quad t \geq 0
$$

Here, $u \geq 0$ is the initial surplus, and $\{S(t)\}$ is a compound Poisson process with positive and negative jumps. We prefer the alternative but mathematically equivalent formulation

$$
U(t)=u-S_{1}(t)+S_{2}(t), \quad t \geq 0 .
$$


Here, $\left\{S_{1}(t)\right\}$ and $\left\{S_{2}(t)\right\}$ are independent compound Poisson processes, each with positive jumps only. The first represents the aggregate claims; it is given by the Poisson parameter $\lambda$ and the probability density function $p(x), x \geq 0$. The second is given by the Poisson parameter $\nu$ and the probability density function $q(x), x \geq 0$. We interpret $S_{2}(t)$ as the aggregate income, which includes premiums and gains. We prefer not to have a term with deterministic premium income $c t$ in (2.1). This facilitates some of the calculations, and the case with deterministic premiums can always be retrieved as a limiting case.

We introduce the discounted penalty function $\phi(u)$ in this model. The penalty at ruin is given by a penalty function $w(x), x \geq 0$ : if the deficit at ruin is $x$, the penalty is $w(x)$. In the following, $\delta>0$ is a constant force for discounting the penalty, and $T$ is the time of ruin. Then

$$
\phi(u)=E\left[e^{-\delta T} w(-U(T)) I(T<\infty) \mid U(0)=u\right]
$$

is the expectation of the discounted penalty at ruin, considered as a function of the initial surplus $u$.

Let $h>0$. By distinguishing according to the time and the amount of the first jump before time $h$ (if there is such a jump), we see by conditioning that

$$
\begin{aligned}
\phi(u)= & \lambda \int_{0}^{h} e^{-(\lambda+\nu+\delta) t} \int_{0}^{u} \phi(u-x) p(x) d x d t+\lambda \int_{0}^{h} e^{-(\lambda+\nu+\delta) t} \int_{u}^{\infty} w(x-u) p(x) d x d t \\
& +\nu \int_{0}^{h} e^{-(\lambda+\nu+\delta) t} \int_{0}^{\infty} \phi(u+x) q(x) d x d t+e^{-(\lambda+\nu+\delta) h} \phi(u) .
\end{aligned}
$$

We differentiate this equation with respect to $h$ and set $h=0$ in the resulting equation. This yields the equation

$$
\lambda \int_{0}^{u} \phi(u-x) p(x) d x+\lambda \int_{u}^{\infty} w(x-u) p(x) d x
$$




$$
+\nu \int_{0}^{\infty} \phi(u+x) q(x) d x-(\lambda+\nu+\delta) \phi(u)=0
$$

(see also equation (4.2) of Labbé and Sendova (2009)). The function $\phi(u)$ can be characterized as the unique solution of this integral equation. To see this, consider the mapping

$$
\begin{gathered}
\phi(u) \rightarrow \quad \frac{\lambda}{\lambda+\nu+\delta} \int_{0}^{u} \phi(u-x) p(x) d x+\frac{\lambda}{\lambda+\nu+\delta} \int_{u}^{\infty} w(x-u) p(x) d x \\
+\frac{\nu}{\lambda+\nu+\delta} \int_{0}^{\infty} \phi(u+x) q(x) d x,
\end{gathered}
$$

which is a contraction and has a unique fixed point. This is based on the Contraction Mapping Theorem, which can be found in many textbooks, for example Burden and Faires (1989).

\section{Combination of exponentials}

We make the additional assumption that the claim size distribution is a combination of $n$ exponential distributions,

$$
p(x)=\sum_{i=1}^{n} A_{i} \beta_{i} e^{-\beta_{i} x}, \quad x>0,
$$

where $\beta_{1}<\beta_{2}<\ldots<\beta_{n}$ and $A_{1}+\ldots+A_{n}=1$. This differs from a mixture, as some of the $A_{i}^{\prime} s$ can be negative as long as $p(x) \geq 0$. For the time being, no restriction is imposed on $q(x)$. Then the discounted penalty function is of the form

$$
\phi(u)=\sum_{k=1}^{n} C_{k} e^{-r_{k} u}, \quad u \geq 0
$$

Indeed, the function (3.2) satisfies equation (2.3), if $r_{1}, \ldots, r_{n}, C_{1}, \ldots, C_{n}$ are properly chosen. To obtain the conditions for these $2 n$ coefficients, we substitute (3.1) and (3.2) 
in (2.3). In the resulting equation, we compare the coefficients of $e^{-r_{k} u}$ to see that $r_{1}, \ldots, r_{n}$ must be solutions of the equation

$$
\lambda \sum_{i=1}^{n} A_{i} \frac{\beta_{i}}{\beta_{i}-r}+\nu \int_{0}^{\infty} e^{-r x} q(x) d x-(\lambda+\nu+\delta)=0 .
$$

This is a generalized Lundberg's equation. The proof in Section 2 of Zhang et al. (2010) can be adapted to show that (3.3) has exactly $n$ solutions with a positive real part (the latter property is necessary in (3.2) because $\phi(u) \rightarrow 0$ for $u \rightarrow \infty$ ). One of these solutions is real-valued, say $r_{1}=R$, with $0<R<\beta_{1}$. The other $n-1$ solutions $r_{2}, \ldots, r_{n}$ have a real part exceeding $R$. Hence, the first term of the sum in (3.2) is dominating for $u \rightarrow \infty$. We exclude the unlikely case where some of the $r_{k}^{\prime}$ s coincide. A comparison of the coefficients of $e^{-\beta_{i} u}$ yields the condition

$$
\sum_{k=1}^{n} \frac{C_{k}}{\beta_{i}-r_{k}}=\frac{\Pi_{i}}{\beta_{i}}, \quad i=1, \ldots, n,
$$

with the notation

$$
\Pi_{i}=\beta_{i} \int_{0}^{\infty} w(x) e^{-\beta_{i} x} d x
$$

This is a system of $n$ linear equations to determine $C_{1}, \ldots, C_{n}$. Its coefficient matrix is the Cauchy matrix, which has a known inverse. However, we prefer to solve (3.4) by a more direct method. We note that the parameter $\nu$ and the function $q(x)$ do not appear explicitly in (3.4). This explains why (3.4) is formally the same as that in the classical case. The formula corresponding to (3.4) in the classical case can be found, for example, in Gerber et al. (2006) (see formula (42)). 


\section{The trick with the rational function}

We define a rational function $Q(r)$ that is associated to $\phi(u)$ in $(3.2)$ :

$$
Q(r)=\sum_{k=1}^{n} \frac{C_{k}}{r-r_{k}} .
$$

If we know the function $Q(r)$, information concerning $\phi(u)$ can readily be obtained according to the formulas

$$
\begin{aligned}
C_{h} & =\lim _{r \rightarrow r_{h}}\left(r-r_{h}\right) Q(r), \quad h=1, \ldots, n, \\
\phi(0) & =\lim _{r \rightarrow \pm \infty} r Q(r)
\end{aligned}
$$

To verify the latter, observe that $\phi(0)=\sum_{k=1}^{n} C_{k}$. Moreover, if

$$
\hat{\phi}(\xi)=\int_{0}^{\infty} e^{-\xi u} \phi(u) d u=-\sum_{k=1}^{n} \frac{C_{k}}{\xi+r_{k}}, \quad \xi>0
$$

denotes the Laplace transform of the function $\phi(u)$, we have

$$
\hat{\phi}(\xi)=-Q(-\xi), \quad \xi>0
$$

The trick is now to find equivalent and more useful expressions for the function $Q(r)$, and to apply (4.2), (4.3) and (4.5) to these expressions. For this purpose, we note that the function $Q(r)$ is completely determined by the following three properties:

- P1) It is a rational function of the type with a polynomial of degree at most $n-1$ divided by one of degree $n$.

- P2) Its poles are $r_{1}, \ldots, r_{n}$.

- P3) $Q\left(\beta_{i}\right)=\frac{\Pi_{i}}{\beta_{i}}, \quad i=1, \ldots, n$, according to $(3.4)$. 
As a first application of this idea, we define the rational function

$$
Q_{1}(r)=\frac{\sum_{j=1}^{n} \frac{\Pi_{j}}{\beta_{j}} \prod_{k=1}^{n}\left(\beta_{j}-r_{k}\right) \prod_{i=1, i \neq j}^{n} \frac{r-\beta_{i}}{\beta_{j}-\beta_{i}}}{\prod_{k=1}^{n}\left(r-r_{k}\right)}
$$

This function satisfies properties P1) - P3) above, which shows that $Q_{1}(r)=Q(r)$. Applying (4.2) to $Q_{1}(r)$, we find that

$$
C_{h}=\frac{\sum_{j=1}^{n} \frac{\Pi_{j}}{\beta_{j}} \prod_{k=1}^{n}\left(\beta_{j}-r_{k}\right) \prod_{i=1, i \neq j}^{n} \frac{r_{h}-\beta_{i}}{\beta_{j}-\beta_{i}}}{\prod_{k=1, k \neq h}^{n}\left(r_{h}-r_{k}\right)}, \quad h=1, \ldots, n
$$

which is the solution of the system of linear equations (3.4). In the following we shall consider the special case where $q(x)$ is exponential, in order to obtain more attractive results.

\section{$5 \quad$ Exponential gains}

In this and the next sections we consider the special case where $q(x)=\alpha e^{-\alpha x}$ for some $\alpha>0$. Then (3.3) becomes the equation

$$
\lambda \sum_{i=1}^{n} A_{i} \frac{\beta_{i}}{\beta_{i}-r}+\nu \frac{\alpha}{\alpha+r}-(\lambda+\nu+\delta)=0 .
$$

This equation has exactly $n+1$ solutions, namely $r_{1}, \ldots, r_{n}$ (introduced earlier) and a unique negative solution $-\rho$. Consider now the rational function

$$
Q_{2}(r)=\frac{\lambda}{\alpha+r} \frac{(\alpha+r) \sum_{i=1}^{n} A_{i} \frac{\Pi_{i}}{\beta_{i}-r}-(\alpha-\rho) \sum_{i=1}^{n} A_{i} \frac{\Pi_{i}}{\beta_{i}+\rho}}{\lambda \sum_{i=1}^{n} A_{i} \frac{\beta_{i}}{\beta_{i}-r}+\nu \frac{\alpha}{\alpha+r}-(\lambda+\nu+\delta)} .
$$


We note that $r=-\rho$ is a common zero of the numerator and the denominator. With this, one verifies that conditions P1) - P3) are satisfied, from which it follows that $Q_{2}(r)=Q(r)$. From (4.2) and L'Hospital's rule we get

$$
C_{h}=\frac{\lambda}{\alpha+r_{h}} \frac{\left(\alpha+r_{h}\right) \sum_{i=1}^{n} A_{i} \frac{\Pi_{i}}{\beta_{i}-r_{h}}-(\alpha-\rho) \sum_{i=1}^{n} A_{i} \frac{\Pi_{i}}{\beta_{i}+\rho}}{\lambda \sum_{i=1}^{n} A_{i} \frac{\beta_{i}}{\left(\beta_{i}-r_{h}\right)^{2}}-\nu \frac{\alpha}{\left(\alpha+r_{h}\right)^{2}}}
$$

which is an alternative to (4.7). Furthermore, an application of (4.3) leads to

$$
\phi(0)=\frac{\lambda}{\lambda+\nu+\delta}\left[\sum_{i=1}^{n} A_{i} \Pi_{i}+(\alpha-\rho) \sum_{i=1}^{n} A_{i} \frac{\Pi_{i}}{\beta_{i}+\rho}\right]
$$

which simplifies to

$$
\phi(0)=\frac{\lambda}{\lambda+\nu+\delta} \sum_{i=1}^{n} A_{i} \Pi_{i} \frac{\beta_{i}+\alpha}{\beta_{i}+\rho} .
$$

\section{General claim amount distributions}

Because any claim amount distribution can be obtained as a limit from an appropriate sequence of combinations of exponential distributions (see for example, Dufresne (2007)), certain results of Section 5 lead to results for an arbitrary $p(x)$. The recipe is simple: rewrite the results in terms of $p(x)$ and $w(x)$, instead of $A_{i}, \beta_{i}, \Pi_{i}$. For example, by observing that for $r<\beta_{1}$

$$
\begin{aligned}
\sum_{i=1}^{n} A_{i} \frac{\Pi_{i}}{\beta_{i}-r} & =\sum_{i=1}^{n} A_{i} \int_{0}^{\infty} \beta_{i} w(x) e^{-\beta_{i} x} d x \int_{0}^{\infty} e^{-\left(\beta_{i}-r\right) y} d y \\
& =\sum_{i=1}^{n} \int_{0}^{\infty} w(x) A_{i} \beta_{i} \int_{0}^{\infty} e^{r y} e^{-\beta_{i}(x+y)} d y d x \\
& =\int_{0}^{\infty} w(x) \int_{0}^{\infty} e^{r y} p(x+y) d y d x
\end{aligned}
$$




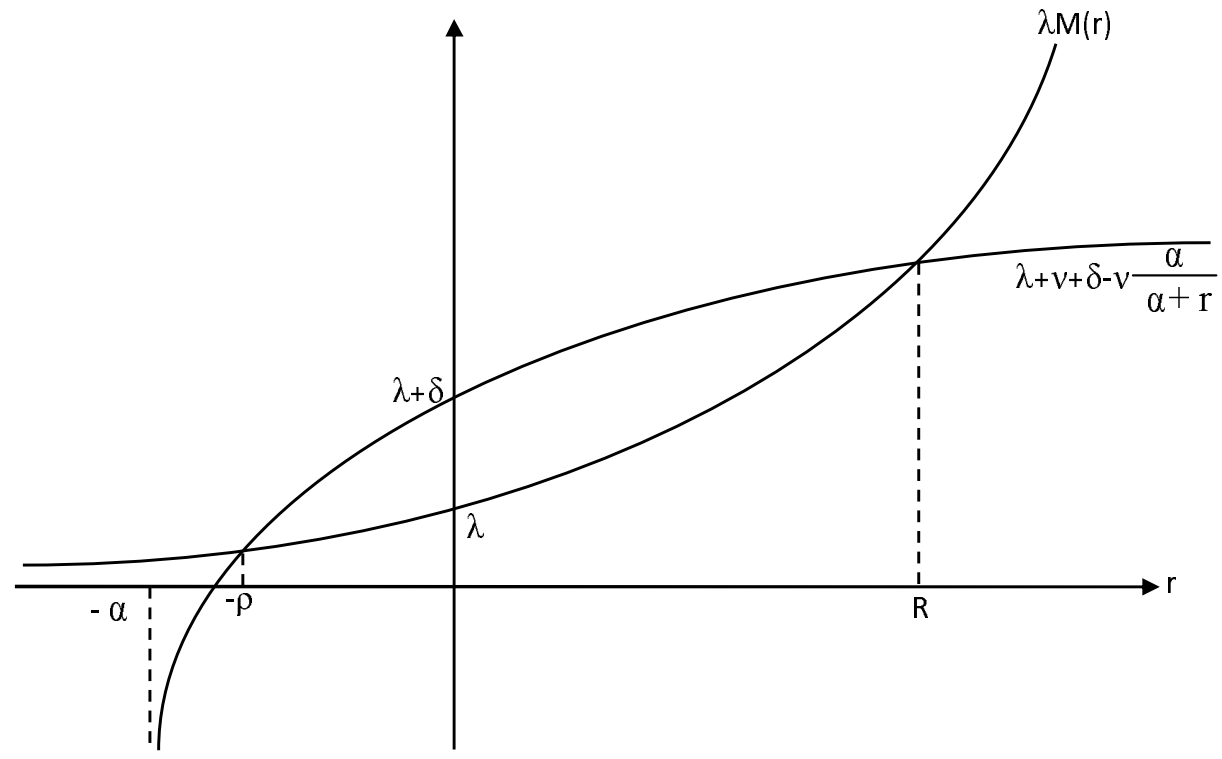

Figure 1: The zeros of the Lundberg function

we can rewrite (5.4) as

$$
\phi(0)=\frac{\lambda}{\lambda+\nu+\delta}\left[\int_{0}^{\infty} w(x) p(x) d x+(\alpha-\rho) \int_{0}^{\infty} w(x) \int_{0}^{\infty} e^{-\rho y} p(x+y) d y d x\right] .
$$

Here $-\rho$ is now the negative zero of the Lundberg function

$$
L(r)=\lambda M(r)+\nu \frac{\alpha}{\alpha+r}-(\lambda+\nu+\delta),
$$

where $M(r)=\int_{0}^{\infty} e^{r x} p(x) d x$ is the moment generating function of $p(x)$, see Figure 1 . 
The function $L(r)$ is defined for $r$ in the interval where $M(r)$ exists. Note that $\lambda /(\lambda+$ $\nu+\delta)$ is the discounted probability that the surplus process has a downward jump before the first upward jump; this explains the first integral in (6.1). Let $g(x)$ denote the discounted probability density function of the deficit at ruin for initial surplus zero. Because (6.1) holds for arbitrary $w(x)$, it follows that

$$
g(x)=\frac{\lambda}{\lambda+\nu+\delta}\left[p(x)+(\alpha-\rho) \int_{0}^{\infty} e^{-\rho y} p(x+y) d y\right], \quad x>0 .
$$

Formula (5.2) can be rewritten as

$$
Q_{2}(r)=\frac{1}{\alpha+r} \frac{(\alpha+r) N(r)-(\alpha-\rho) N(-\rho)}{L(r)},
$$

with the auxiliary function

$$
N(r)=\lambda \int_{0}^{\infty} w(x) \int_{0}^{\infty} e^{r y} p(x+y) d y d x
$$

and $L(r)$ as given in (6.2). In general, $Q_{2}(r)$ is not a rational function, and its use should be restricted, for example to $r<0$. According to (4.5), the Laplace transform of $\phi(u)$ is

$$
\hat{\phi}(\xi)=\frac{1}{\alpha-\xi} \frac{(\alpha-\rho) N(-\rho)-(\alpha-\xi) N(-\xi)}{L(-\xi)}, \quad \xi>0
$$

for an arbitrary $p(x)$.

We note that (6.1) can be recovered from (6.4) by means of (4.3). For this, it is important to take the limit as $r \rightarrow-\infty$.

Now we turn to formula (5.3). In general, it is not useful unless $h=1$. Assuming the existence of $R$ (the positive zero of $L(r)$ ), we have the asymptotic formula

$$
\phi(u) \sim C e^{-R u} \quad \text { for } u \rightarrow \infty,
$$


with

$$
C=\frac{1}{\alpha+R} \frac{(\alpha+R) N(R)-(\alpha-\rho) N(-\rho)}{L^{\prime}(R)} .
$$

Let us consider the special case where $w(x)=1$. Then $\phi(u)$ is the expected present value of 1 payable at the time of ruin. From (6.1) we have

$$
\phi(0)=\frac{\lambda}{\lambda+\nu+\delta}\left[1+(\alpha-\rho) \int_{0}^{\infty} \int_{0}^{\infty} e^{-\rho y} p(x+y) d y d x\right] .
$$

To evaluate this expression, we use the formula

$$
\int_{0}^{\infty} \int_{0}^{\infty} e^{r y} p(x+y) d y d x=\frac{1}{r}[M(r)-1]
$$

for $r=-\rho$ and the fact that $L(-\rho)=0$. After simplification we find that

$$
\phi(0)=1-\frac{\alpha \delta}{\rho(\lambda+\nu+\delta)} .
$$

The negative term of this surprisingly simple formula shows the effect of discounting and the possibility of survival.

\section{The classical model}

In the classical model, the surplus at time $t$ is $U(t)=u+c t-S_{1}(t)$. This model can be obtained as a limit from the model of Section 6 . For $\nu \rightarrow \infty, \alpha \rightarrow \infty$ such that $\nu / \alpha=c$, we have $S_{2}(t)=c t$ in the limit. By taking this limit, we can retrieve several known results in a straightforward manner.

First, (6.2) becomes

$$
L(r)=\lambda M(r)-c r-(\lambda+\delta)
$$


in the limit. Then $-\rho$ is the negative zero and $R$ (if it exists) the positive zero of $L(r)$. Formula (6.1) leads to

$$
\phi(0)=\frac{\lambda}{c} \int_{0}^{\infty} w(x) \int_{0}^{\infty} e^{-\rho y} p(x+y) d y d x
$$

in the limit, while (6.3) yields

$$
g(x)=\frac{\lambda}{c} \int_{0}^{\infty} e^{-\rho y} p(x+y) d y, \quad x>0 .
$$

This result is indeed formula (3.14) in Gerber and Shiu (1997) and also (3.4) in Gerber and Shiu (1998). From (6.4) we see that $Q_{2}(r)$ can be written as

$$
Q_{2}(r)=\frac{\lambda}{L(r)} \int_{0}^{\infty} w(x) \int_{0}^{\infty}\left(e^{r y}-e^{-\rho y}\right) p(x+y) d y d x .
$$

The resulting formula for $\hat{\phi}(\xi)=-Q_{2}(-\xi)$ is contained in (2.48) together with $(2.52)$ and (2.56) of Gerber and Shiu (1998). Moreover, from (6.8) we get the limiting value

$$
C=\frac{\lambda}{L^{\prime}(R)} \int_{0}^{\infty} w(x) \int_{0}^{\infty}\left(e^{R y}-e^{-\rho y}\right) p(x+y) d y d x
$$

which is formula (4.10) in Gerber and Shiu (1998).

Finally, if $w(x)=1$, formula (6.11) yields

$$
\phi(0)=1-\frac{\delta}{\rho c}
$$

in the limit. This is formula (3.9) in Gerber and Shiu (1998). Furthermore, (7.5) simplifies to

$$
C=\frac{\delta}{L^{\prime}(R)}\left(\frac{1}{R}+\frac{1}{\rho}\right)
$$

To verify this, use (6.10) with $r=R$ and $-\rho$ and that $L(R)=L(-\rho)=0$. 


\section{The probability of ruin}

In the limit $\delta=0$ and the special case $w(x)=1, \phi(u)$ becomes $\psi(u)$, the probability of ruin. We assume a positive loading, that is that $\nu / \alpha>\lambda p_{1}$ in Section 6 and $c>\lambda p_{1}$ in Section 7, where $p_{1}$ denotes the mean claim amount. Then $\rho=0$ in the limit. From (6.1) we see that

$$
\psi(0)=\frac{\lambda}{\lambda+\nu}\left(1+\alpha p_{1}\right)
$$

and (7.2) yields a classical result of ruin theory,

$$
\psi(0)=\frac{\lambda p_{1}}{c}
$$

Of course, this formula can also be obtained as a limit from (8.1). Formula (8.1) can be reformulated as

$$
1-\psi(0)=\frac{\alpha}{\lambda+\nu}\left(\frac{\nu}{\alpha}-\lambda p_{1}\right)
$$

Note that the expression $\nu / \alpha-\lambda p_{1}$ is the expected increase of the surplus per unit time. To obtain (8.3) as a limit from (6.11), observe that $\delta / \rho \rightarrow \nu / \alpha-\lambda p_{1}$ for $\delta \rightarrow 0$. Formula (8.1) can also be reformulated as

$$
\psi(0)=\frac{\lambda}{\lambda+\nu}+\frac{\nu}{\lambda+\nu} \frac{\lambda p_{1}}{\nu / \alpha}=\frac{\lambda}{\lambda+\nu}+\frac{\nu}{\lambda+\nu} \frac{E\left[S_{1}(1)\right]}{E\left[S_{2}(1)\right]},
$$

which has the following interpretation: The first term is the probability that $S_{1}(t)$ has a jump before $S_{2}(t)$, in which case ruin occurs at that time. Given that $S_{2}(t)$ has a jump before $S_{1}(t)$, the probability of which is $\nu /(\lambda+\nu)$, the conditional probability of ruin is $E\left[S_{1}(1)\right] / E\left[S_{2}(1)\right]$. Formula (8.4) appears to be a natural extension of the classical result (8.2). 
From (6.3) we get

$$
g(x)=\frac{\lambda}{\lambda+\nu}[p(x)+\alpha(1-P(x))], \quad x>0,
$$

which reduces to

$$
g(x)=\frac{\lambda}{c}[1-P(x)], \quad x>0 .
$$

in the classical model of Section 7. The latter formula can be found, for example, in Theorem 13.5.1 of Bowers et al. (1997). The determination of the Laplace transform is straightforward. In the classical model of Section 7, we get from (7.1)

$$
\hat{\psi}(\xi)=\frac{\lambda}{L(-\xi)}\left[p_{1}-\int_{0}^{\infty} \int_{0}^{\infty} e^{-\xi y} p(x+y) d y d x\right], \quad \xi>0
$$

with $L(r)=\lambda M(r)-c r-\lambda$. To reconcile (8.7) with formula (2.60) in Gerber and Shiu (1998), use (6.10) with $r=-\xi$.

Finally, we turn to the asymptotic formula, $\psi(u) \sim C e^{-R u}$ for $u \rightarrow \infty$. The coefficient $C$ is obtained as a limit from (6.8). We have $N(0)=\lambda p_{1}$. By using (6.10) with $r=R$ and the fact that $L(R)=0$, we find that $N(R)=\nu /(\alpha+R)$. Hence,

$$
C=\frac{\nu-\lambda \alpha p_{1}}{(\alpha+R) L^{\prime}(R)}
$$

by (6.8). In the classical model of Section 7 , this yields the formula

$$
C=\frac{c-\lambda p_{1}}{L^{\prime}(R)}
$$

which is a famous result of the Scandinavian school. To obtain (8.9) from (7.7), recall that $\delta / \rho \rightarrow c-\lambda p_{1}$ for $\delta \rightarrow 0$. 


\section{Perturbation by diffusion}

The methodology can also be applied, if the surplus process (2.1) is perturbed by an independent diffusion process. The steps are very similar. For this reason we summarize them in a condensed form in a single section and highlight only the necessary modifications. We assume that

$$
U(t)=u-S_{1}(t)+S_{2}(t)+\sigma W(t), \quad t \geq 0,
$$

where $\{W(t)\}$ is an independent standard Wiener process. There are two kinds of ruin in this model, by a claim (which results in a deficit at ruin) or by oscillation. The penalty for the first is given by the function $w(x)$. For the second, it is given by constant $w_{0}$. Instead of (2.3), the discounted penalty function now satisfies the functional equation

$$
\begin{aligned}
\lambda \int_{0}^{u} \phi & (u-x) p(x) d x+\lambda \int_{u}^{\infty} w(x-u) p(x) d x \\
& +\nu \int_{0}^{\infty} \phi(u+x) q(x) d x-(\lambda+\nu+\delta) \phi(u)+D \phi^{\prime \prime}(u)=0
\end{aligned}
$$

with the notation $D=\sigma^{2} / 2$.

As in (3.1), we assume that the claim size distribution is a combination of $n$ exponential distributions. The discounted penalty function $\phi(u)$ is then of the form

$$
\phi(u)=\sum_{k=1}^{n+1} C_{k} e^{-r_{k} u}, \quad u \geq 0 .
$$

To determine $r_{1}, \ldots, r_{n+1}, C_{1}, \ldots, C_{n+1}$, we substitute (9.3) in (9.2) and find that $r_{1}, \ldots, r_{n+1}$ are the $n+1$ solutions with positive real part of the equation

$$
\lambda \sum_{i=1}^{n} A_{i} \frac{\beta_{i}}{\beta_{i}-r}+\nu \int_{0}^{\infty} e^{-r x} q(x) d x-(\lambda+\nu+\delta)+D r^{2}=0,
$$


and $C_{1}, \ldots, C_{n+1}$ are solutions of the following system of $n+1$ equations:

$$
\begin{aligned}
\sum_{k=1}^{n+1} \frac{C_{k}}{\beta_{i}-r_{k}} & =\frac{\Pi_{i}}{\beta_{i}}, \quad i=1, \ldots, n, \\
C_{1}+\ldots+C_{n+1} & =w_{0} .
\end{aligned}
$$

The latter condition follows from $\phi(0)=w_{0}$. We note that the solutions of (9.4) are analyzed in Zhang et al. (2010).

In analogy to (4.1), we define the rational function $Q(r)$ as

$$
Q(r)=\sum_{k=1}^{n+1} \frac{C_{k}}{r-r_{k}}
$$

Knowing this function, we can obtain

$$
C_{h}=\lim _{r \rightarrow r_{h}}\left(r-r_{h}\right) Q(r), \quad h=1, \ldots, n+1,
$$

and

$$
\hat{\phi}(\xi)=-Q(-\xi), \quad \xi>0
$$

the Laplace transform of $\phi(u)$. The function $Q(r)$ is characterized by the following four properties:

- P1) It is a rational function of the type with a polynomial of degree at most $n$ divided by one of degree $n+1$.

- P2) Its poles are $r_{1}, \ldots, r_{n+1}$.

- P3) $Q\left(\beta_{i}\right)=\frac{\Pi_{i}}{\beta_{i}}, \quad i=1, \ldots, n$, according to $(9.5)$.

- P4) $\lim _{r \rightarrow \pm \infty} r Q(r)=w_{0}$, according to (9.6). 
These properties are satisfied by the rational function

$$
Q_{1}(r)=\frac{\sum_{j=1}^{n} \frac{\Pi_{j}}{\beta_{j}} \prod_{k=1}^{n+1}\left(\beta_{j}-r_{k}\right) \prod_{i=1, i \neq j}^{n} \frac{r-\beta_{i}}{\beta_{j}-\beta_{i}}+w_{0} \prod_{i=1}^{n}\left(r-\beta_{i}\right)}{\prod_{k=1}^{n+1}\left(r-r_{k}\right)},
$$

and hence $Q_{1}(r)=Q(r)$. Application of (9.8) yields

$$
C_{h}=\frac{\sum_{j=1}^{n} \frac{\Pi_{j}}{\beta_{j}} \prod_{k=1}^{n+1}\left(\beta_{j}-r_{k}\right) \prod_{i=1, i \neq j}^{n} \frac{r_{h}-\beta_{i}}{\beta_{j}-\beta_{i}}+w_{0} \prod_{i=1}^{n}\left(r_{h}-\beta_{i}\right)}{\prod_{k=1, k \neq h}^{n+1}\left(r_{h}-r_{k}\right)}, \quad h=1, \ldots, n+1 .
$$

As in Section 5, we make the additional assumption that $q(x)=\alpha e^{-\alpha x}$. Then (9.4) reduces to the equation

$$
\lambda \sum_{i=1}^{n} A_{i} \frac{\beta_{i}}{\beta_{i}-r}+\nu \frac{\alpha}{\alpha+r}-(\lambda+\nu+\delta)+D r^{2}=0
$$

This is equivalent to a polynomial equation of degree $n+3$. Its $n+3$ solutions are $r_{1}, \ldots, r_{n+1}$ with a positive real part and the two negative solutions $-\rho$ and $-\rho_{d}$ with $-\infty<-\rho_{d}<-\alpha<-\rho<0$. Then the function

$$
Q_{2}(r)=\frac{1}{\alpha+r} \frac{\lambda(\alpha+r) \sum_{i=1}^{n} A_{i} \frac{\Pi_{i}}{\beta_{i}-r}-\lambda(\alpha-\rho) \sum_{i=1}^{n} A_{i} \frac{\Pi_{i}}{\beta_{i}+\rho}+D w_{0}(r+\rho)(r+\kappa)}{\lambda \sum_{i=1}^{n} A_{i} \frac{\beta_{i}}{\beta_{i}-r}+\nu \frac{\alpha}{\alpha+r}-(\lambda+\nu+\delta)+D r^{2}}
$$

satisfies properties $\mathrm{P} 1)-\mathrm{P} 4)$, provided that $\kappa$ is chosen such that the numerator vanishes for $r=-\rho_{d}$.

For an arbitrary $p(x)$, the function $Q_{2}(r)$ can be written as

$$
Q_{2}(r)=\frac{1}{\alpha+r} \frac{(\alpha+r) N(r)-(\alpha-\rho) N(-\rho)+D w_{0}(r+\rho)(r+\kappa)}{L(r)}
$$




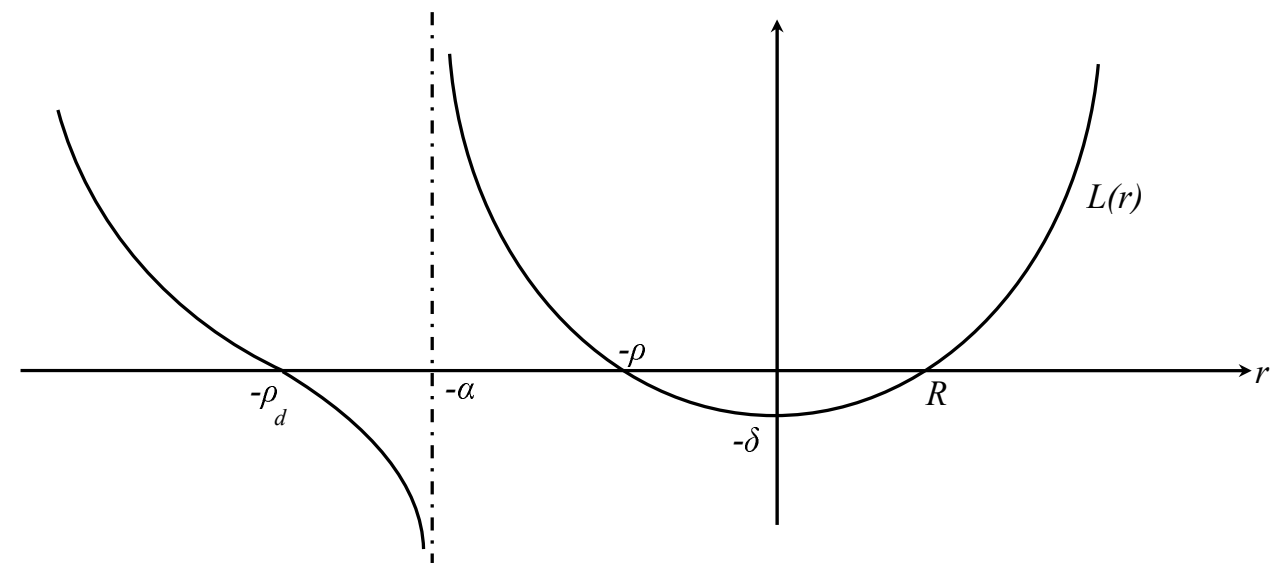

Figure 2: The zeros of $L(r)$

where $N(r)$ is defined as in (6.5),

$$
\kappa=\rho_{d}+\frac{(\alpha-\rho) N(-\rho)-\left(\alpha-\rho_{d}\right) N\left(-\rho_{d}\right)}{D w_{0}\left(\rho-\rho_{d}\right)},
$$

and

$$
L(r)=\lambda M(r)+\nu \frac{\alpha}{\alpha+r}-(\lambda+\nu+\delta)+D r^{2} .
$$

The zeros of $L(r)$ are illustrated by Figure 2 .

From (9.13) or (9.14) combined with (9.8) and (9.9), expressions for the Laplace transform and the asymptotic formula of $\phi(u)$ can be calculated in a straightforward manner.

As in Section 7, we consider the model with deterministic premium ct, that is now

$$
U(t)=u+c t-S_{1}(t)+\sigma W(t) .
$$


This is the model examined by Gerber and Landry (1998). Now

$$
Q_{2}(r)=\frac{\lambda \sum_{i=1}^{n} A_{i} \frac{\Pi_{i}}{\beta_{i}-r}-\lambda \sum_{i=1}^{n} A_{i} \frac{\Pi_{i}}{\beta_{i}+\rho}+D w_{0}(r+\rho)}{\lambda \sum_{i=1}^{n} A_{i} \frac{\beta_{i}}{\beta_{i}-r}-c r-(\lambda+\delta)+D r^{2}}
$$

does the job, that is $Q_{2}(r)=Q(r)$. It is possible to show that the function (9.13) converges to the function (9.18) if $\alpha \rightarrow \infty, \nu \rightarrow \infty$ with $\nu / \alpha=c$. For this one has to show that $\rho_{d} / \alpha \rightarrow 1$, if $\alpha \rightarrow \infty$. This is proved in the Appendix.

For an arbitrary $p(x),(9.18)$ becomes

$$
Q_{2}(r)=\frac{N(r)-N(-\rho)+D w_{0}(r+\rho)}{L(r)},
$$

with $L(r)$ given by

$$
L(r)=\lambda M(r)-c r-(\lambda+\delta)+D r^{2},
$$

$N(r)$ defined as in (6.5), and $-\rho$ the negative zero of $L(r)$. From (9.9) we obtain the Laplace transform of $\phi(u)$ :

$$
\hat{\phi}(\xi)=\frac{N(-\rho)-N(-\xi)-D w_{0}(\rho-\xi)}{L(-\xi)}, \quad \xi>0 .
$$

From (9.8) with $h=1\left(r_{1}=R\right)$, we obtain

$$
C=\frac{N(R)-N(-\rho)+D w_{0}(R+\rho)}{L^{\prime}(R)},
$$

which is needed in the asymptotic formula for $\phi(u)$. Let us consider the special case $w(x)=w_{0}=1$. Then (9.22) simplifies to

$$
C=\frac{\delta}{L^{\prime}(R)}\left(\frac{1}{R}+\frac{1}{\rho}\right)
$$


To see this, use (6.10) for $r=R$ and $r=-\rho$ and remember that $L(R)=L(-\rho)=0$. Note that (9.23) is formally the same as (7.7). Of course the $D$ is contained implicitly in $R, \rho$ and $L^{\prime}(R)$.

To obtain results for the probability of ruin, we assume $c>\lambda p_{1}$ and take the limit $\delta \rightarrow 0$. Then $\rho \rightarrow 0$ and $\delta / \rho \rightarrow c-\lambda p_{1}$. We take the limit in (9.22) and see that in the asymptotic formula,

$$
\psi(u) \sim C e^{-R u} \quad \text { for } u \rightarrow \infty,
$$

the coefficient $C$ has formally the same value as in (8.9). The Laplace transform of $\psi(u)$ is

$$
\hat{\psi}(\xi)=\frac{N(0)-N(-\xi)+D \xi}{L(-\xi)}, \quad \xi>0
$$

with

$$
\begin{aligned}
L(-\xi) & =\lambda M(-\xi)+c \xi-\lambda+D \xi^{2}, \\
N(0) & =\lambda p_{1} \\
N(-\xi) & =-\frac{\lambda}{\xi}[M(-\xi)-1] .
\end{aligned}
$$

As a check, set $\lambda=0$. Then (9.24) reduces to

$$
\hat{\psi}(\xi)=\frac{D}{c+D \xi}=\frac{1}{R+\xi}, \quad \xi>0,
$$

with $R=c / D$. This is indeed the Laplace transform of the well-known expression $\psi(u)=e^{-R u}$ for a diffusion process. For another check, consider the limit $D \rightarrow 0$ in (9.24). We obtain the well-known expression for Laplace transform of the probability of ruin in the classical model, see, for example (2.60) in Gerber and Shiu (1998), or formula (2.9) in Chapter XIV.3 of Feller (1971). 
As in Dufresne and Gerber (1991), the probability of ruin is decomposed as

$$
\psi(u)=\psi_{s}(u)+\psi_{d}(u)
$$

the probability of ruin by a claim plus the probability of ruin by oscillation. (The index $s$ is from the French word for claim, sinistre, and $d$ stands for diffusion). To obtain $\psi_{d}(u)$, we set $w(x)=0, w_{0}=1$. This leads to

$$
\hat{\psi}_{d}(\xi)=\frac{D \xi}{L(-\xi)}, \quad \xi>0
$$

For the asymptotic formula $\psi_{d}(u) \sim C_{d} e^{-R u}, u \rightarrow \infty$, we find

$$
C_{d}=\frac{D R}{L^{\prime}(R)}
$$

From (9.24) and (9.26) it follows that

$$
\hat{\psi}_{s}(\xi)=\frac{N(0)-N(-\xi)}{L(-\xi)}, \quad \xi>0 .
$$

Furthermore $C_{s}=C-C_{d}$ for the asymptotic formula. The expressions for $C, C_{d}$ and $C_{s}$ can be found as formulas (7.30) - (7.32) in Dufresne (1989).

One should note that the diffusion perturbation $W(t)$ in (9.1) could itself be obtained as a limit of a family of independent compound Poisson processes, see for instance Sarkar and Sen (2005).

\section{Concluding Remarks}

This paper presents a new and in some sense elementary and pedagogical approach to obtain a series of results for the discounted penalty function. The method could be 
applied directly in the classical model of collective risk theory. However, we prefer to explain the method in a model where the deterministic premium income is replaced by an independent compound Poisson process with exponentially distributed jumps, because this more advanced model can be treated with the same direct approach and it contains the classical model as a limit. Also, because there are upward and downward jumps in this model, the results may have applications in finance, in particular the pricing of barrier and other American options. Finally, we note that this more general model facilitates a simple interpretation of the dividends-penalty identity, again with the classical identity as a natural limit, see Gerber and Yang (2010).

The method consists of two steps. In the first step, results are obtained for the special case where the claim amount distribution is a combination of exponential distributions. This family is dense in the set of all claim amount distributions. Since the discounted penalty function can be expressed solely through the negative root of the generalized Lundberg equation, one can then in a second step express the results in general terms to obtain the results for an arbitrary claim amount distribution. This recipe works for all sufficiently well-behaved penalty functions and in particular for all those of practical interest. The method can in principle be extended to the case where the distribution of the upward jumps is a combination of $m$ exponential distributions. Then, the resulting expression depends on the $m$ zeros with negative real part of the generalized Lundberg equation, and the results are not nearly as elegant as in the case $m=1$. 


\section{Appendix: Proof of $\rho_{d} / \alpha \rightarrow 1$}

Let $r_{1}, \ldots, r_{n+1},-\rho$ be the solutions of the equation

$$
\lambda \sum_{i=1}^{n} A_{i} \frac{\beta_{i}}{\beta_{i}-r}-c r-(\lambda+\delta)+D r^{2}=0 .
$$

By Vieta's rule (for the product of the solutions of a polynomial equation) we have

$$
\rho \prod_{k=1}^{n+1} r_{k}=\delta \prod_{i=1}^{n} \beta_{i} / D
$$

Now, let $r_{1}(\alpha), \ldots, r_{n+1}(\alpha),-\rho(\alpha),-\rho_{d}(\alpha)$ denote the solutions of the equation

$$
\lambda \sum_{i=1}^{n} A_{i} \frac{\beta_{i}}{\beta_{i}-r}+\nu \frac{\alpha}{\alpha+r}-(\lambda+\nu+\delta)+D r^{2}=0,
$$

with $\nu / \alpha=c$, such that $r_{k}(\alpha) \rightarrow r_{k}$ and $\rho(\alpha) \rightarrow \rho$ for $\alpha \rightarrow \infty$. Using again Vieta's rule and (A.2) we see that

$$
\rho(\alpha) \rho_{d}(\alpha) \prod_{k=1}^{n+1} r_{k}(\alpha)=\alpha \delta \prod_{i=1}^{n} \beta_{i} / D=\alpha \rho \prod_{k=1}^{n+1} r_{k} .
$$

Now divide this equation by $\alpha$ and let $\alpha \rightarrow \infty$ to see that $\rho_{d}(\alpha) / \alpha \rightarrow 1$ for $\alpha \rightarrow \infty$. This shows that (9.18) follows from (9.13) as a limit.

\section{Acknowledgement}

The authors would like to thank two anonymous referees for their constructive remarks. Hansjörg Albrecher gratefully acknowledges the financial support from the Swiss National Science Foundation Project 200021-124635/1. Hailiang Yang would like 
to acknowledge the Research Grants Council of the Hong Kong Special Administrative Region, China (project No. HKU 7540/08H).

\section{REFERENCES}

Asmussen, Soren. Madan, Dilip and Pistorius, Martijn R. 2008. "Pricing equity default swaps under an approximation to the CGMY Lévy model," Journal of Computational Finance, 11, 79-93.

BAO, Then-Hua. 2006. "The expected discounted penalty at ruin in the risk process with random income," Applied Mathematics and Computation, 179, 559-566.

BoucherIe, Richard J. and Onno J. Boxma. 1996. "The workload in the M/G/1 queue with work removal," Probability in Engineering and Informational Systems, 10, 261-277.

Boucherie, Richard J., Onno J. Boxma and Karl Sigman. 1997. "A note on negative customers, GI/G/1 workload, and risk processes," Probability in Engineering and Informational Systems, 11, 305-311.

Bowers, Newton L., Hans U. Gerber, James C. Hickman, Donald A. Jones and Cecil J. Nesbitt. 1997. Actuarial Mathematics, Second Edition. The Society of Actuaries: Schaumburg, IL.

Burden, Richard L. and J. Douglas FaIRes. 1989. Numerical Analysis, Fourth Edition. PWS-Kent Publishing Company. 
Cai, Jun, Runhuan Feng and Gordon E. Willmot. 2009. "On the expectation of total discounted operating costs up to default and its applications," Advances in Applied Probability, 41, 495-522.

Cramér, Harald. 1955. Collective Risk Theory: A Survey from the Point of View of Stochastic Processes, Skandia Jubilee Volume.

Dufresne, Daniel. 2007. "Fitting combinations of exponentials to probability distributions," Applied Stochastic Models in Business and Industry, 23(1): 23-48.

Dufresne, François. 1989. Probabilité et sévérité de la ruine: Modéle classique de la théorie du risque et une de ses extensions, Doctoral thesis, University of Lausanne.

Dufresne, François and Hans U. Gerber. 1989. "Three methods to calculate the probability of ruin," ASTIN Bulletin, 19(1): 71-90.

Dufresne, François and Hans U. Gerber. 1991. "Risk theory for the compound Poisson process that is perturbed by diffusion," Insurance: Mathematics and Economics, 10: 51-59.

Feller, William. 1971. An Introduction to Probability Theory and Its Applications, John Wiley \& Sons, New York.

Gerber, Hans U. and Elias S. W. Shiu. 1997. "The joint distribution of the time of ruin, the surplus immediately before ruin, and the deficit at ruin," Insurance: Mathematics and Economics, 21: 129-137.

Gerber, Hans U. and Elias S. W. Shiu. 1998. "On the time value of ruin," North American Actuarial Journal, 2(1): 48-78. 
Gerber, Hans U. and Bruno Landry. 1998. "On the discounted penalty at ruin in a jump-diffusion and the perpetual put option," Insurance: Mathematics and Economics, 22(3): 263-276.

Gerber, Hans U., Sheldon, X. Lin and Hailiang YAng. 2006. "A Note on the Dividends-penalty Identity and the Optimal Dividend Barrier," ASTIN Bulletin, 36(2): 489-503.

Gerber, Hans U. and Hailiang YAng. 2010. "Obtaining the dividends-penalty identities by interpretation," Insurance: Mathematics and Economics, in press.

Kou, Steve and Hui Wang. 2003. "First passage times of a jump diffusion process," Advances in Applied Probability, 35, 504-531.

JACOBSEn, Martin. 2005. "The time to ruin for a class of Markov additive risk process with two-sided jumps," Advances in Applied Probability, 37, 963-992.

LABBÉ, Chantal and Kristina Sendova. 2009. "The expected discounted penalty function under a risk model with stochastic income," Applied Mathematics and Computation, 215, 1852-1867.

Levendorskit, Sergei Z. 2004. "Pricing of the American put under Lévy processes," International Journal of Theoretical and Applied Finance, 7(3), 303-335.

LEwis, Alan L. and Ernesto Mordecki. 2008. "Wiener-Hopf factorization for Lévy processes having positive jumps with rational transforms," Journal of Applied Probability, 45(1), 118-134.

Perry, David, Wolfgang Stadje and S. Zacks. 2002. "First-exit times for compound Poisson processes for some types of positive and negative jumps," Stochas- 
tic Models, 18(1): 139-157.

SARKAR, Joykrishna and Arusharka SEn. 2005. "Weak convergence approach to compound Poisson risk processes perturbed by diffusion," Insurance: Mathematics and Economics, 36(3): 421-432.

Schmidui, Hanspeter. 2010. "On the Gerber-Shiu function and change of measure," Insurance: Mathematics and Economics, 46(1): 3-11.

Segerdahl, Carl Otto. 1939. On Homogeneous Random Processes and Collective Risk Theory, Thesis, Stockholm.

Temnov, Gregory. 2004. "Risk process with random income," Journal of Mathematical Sciences, 123(1), 3780-3794.

Xing, Xiaoyu, Wei Zhang and Yiming Jiang. 2008. "On the time to ruin and the deficit at ruin in a risk model with double-sided jumps," Statistics and Probability Letters, 78, 2692-2699.

ZHANG, Zhimin, Hu YANG and Shuanming LI. 2010. "The perturbed compound Poisson risk model with two-sided jumps," Journal of Computational and Applied Mathematics, 233: 1773-1784.

ZHANG, Zhimin and Hu YANG. 2010. "A generalized penalty function in the Sparre Andersen risk model with two-sided jumps," Statistics and Probability Letters, 80(7-8): 597-607. 